Original Research Paper

\title{
Antibody Responses to HIV-1 gp120 Hypervariable Regions in Six Long-Term Non-Progressors
}

\author{
${ }^{1}$ Rebecca Rivera, ${ }^{1}$ Kyung Hee Kang, ${ }^{2}$ Murray B. Gardner, ${ }^{1}$ David E. Anderson, ${ }^{3}$ Santiago Collado-Chastel, \\ ${ }^{4}$ Eddy Rios-Olivares, ${ }^{5}$ Yasuhiro Yamamura, ${ }^{6}$ Francisco Diaz-Mitoma, ${ }^{1} \mathrm{Xia}$ Li and ${ }^{1}$ José V. Torres \\ ${ }^{I}$ Department of Medical Microbiology and Immunology, School of Medicine, University of California, Davis, California, USA \\ ${ }^{2}$ Department of Medical Pathology and Laboratory Medicine and Center of Comparative Medicine, School of Medicine, \\ University of California, Davis, California, USA \\ ${ }^{3}$ Institute of Biomedical Studies, Santo Domingo, Dominican Republic \\ ${ }^{4}$ Department of Microbiology and Immunology, Universidad Central del Caribe, Bayamon, PR, USA \\ ${ }^{5}$ Department of Microbiology, Ponce School of Medicine, Ponce, PR, USA \\ ${ }^{6}$ Advanced Medical Research Institute of Canada (AMRIC), 41 Ramsey Lake Road, Sudbury, ON Canada
}

Article history

Received: 07-08-2014

Revised: $15-08-2015$

Accepted: 27-06-2016

Corresponding Author: José V. Torres

Department of Medical Microbiology and

Immunology, School of

Medicine, University of

California, Davis, California,

USA

E-mail: jvtorres@ucdavis.edu

\begin{abstract}
Our long-term goal is to discover the combination of host parameters that help some HIV-infected individuals to resist progression to AIDS. In this study, we examined antibody responses using multiple samples obtained from a cohort of Long-Term NonProgressors (LTNPs). Our hypothesis is that antibody responses to variable regions of the HIV-1 envelope glycoprotein are involved in reducing the viral load associated with LTNPs and that these specific immune responses influence susceptibility to disease progression. Multiple plasma samples were obtained from patients identified as LTNPs with the objective of characterizing humoral immune response directed to the five hypervariable regions of the envelope glycoprotein. Antibody binding was tested against peptides representing the five hypervariable regions of gp120, as well as against analog peptides representing different isolates of HIV-1 and against recombinant envelope glycoprotein. LTNPs have specific antibodies to the hypervariable regions of the envelope glycoprotein and develop different patterns of antibody recognition to variable epitopes of envelope glycoprotein. These antibodies can be detected using HIV peptides as capture antigens.
\end{abstract}

Keywords: HIV-1, Envelope, Peptide, Long-Term Non-Progressors, Humoral Immune Response

\section{Introduction}

The envelope glycoprotein (Env) of HIV-1 is formed by heterodimers in a trimeric configuration, consisting of three gp120 surface envelope glycoproteins noncovalently bound to three gp41 transmembrane glycoproteins (Helseth et al., 1991; Wyatt and Sodroski, 1998). Each gp120 monomer contains five hypervariable regions (V1-V5) separated by five constant regions (C1C4) (Modrow et al., 1987). The gp120 surface envelope glycoprotein of HIV-1 mediates viral attachment to target cells and elicits antibodies that neutralize HIV infection (Pantophlet and Burton, 2006). When gp120 binds to the cellular CD4 receptor, it undergoes conformational changes that expose the binding site of gp120 for chemokine co-receptors, including CCR5 or CXCR4 (Feng et al., 1996).
The third hypervariable region (V3) is considered the major viral determinant of co-receptor specificity due to the fact that the co-receptor binding site resides primarily in the V3 region following conformational changes induced by binding to CD4. However, it is clear that V1, $\mathrm{V} 2$ and other regions are also involved (Speck et al., 1997; Kwong et al., 1998; Jensen and van't Wout, 2003). The V3 region also contains antigenic epitopes that induce both cellular and humoral immune responses, including neutralizing antibodies (Fomsgaard et al., 1998; Cardozo et al., 2009; Swetnam et al., 2010).

The V1 and V2 regions are also highly variable in polymorphism, genetic sequence and N-linked glycosylation sites, factors that may affect attachment of gp120 to target cells, the usage of co-receptors and recognition by neutralizing antibodies (Hughes and Bell,
Science

Publications 
1997; Curlin et al., 2010). The V1/V2 regions affect the sensitivity to neutralizing antibodies and evolve under positive natural selection in vivo (Sagar et al., 2006). Studies have found that V1/V2 regions elongates during chronic infection as well as in Long-Term NonProgressors (LTNPs), but not within rapid AIDS progressors (Shioda et al., 1997; Kitrinos et al., 2003; Curlin et al., 2010).

Our laboratory has developed an approach to prepare a peptide construct that represents epitope variability in a single synthesis. HIV Hypervariable Epitope Constructs (HECs) are synthetic peptide mixtures that contain multiple variants of a given epitope, based on five variable regions of the gp120 envelope glycoprotein of HIV-1. In previous studies, we assessed the immunogenicity of HIV HECs in rhesus macaques and rabbits (Carlos et al., 2000) and showed that HIV HECs are strong immunogens, eliciting both humoral and cellular immune responses. Our previous work also demonstrated that HIV-1 infected individuals with subtypes A, B, C, D, E and F from many different countries recognize all individual HIV HECs. Moreover, more than $96 \%$ of sera from a cohort $(\mathrm{N}=1194)$ of HIV1 positive and AIDS patients from Puerto Rico and Canada showed antibody reactivity to the HIV HECs (Carlos et al., 1999).

In the present study, we wanted to determine to what extent LTNPs have antibodies that recognize the HIV gp120 hypervariable regions and if their antibody response would change during the time period examined.

\section{Material and Methods}

\section{HIV HEC Design and Synthesis}

Protein sequences corresponding to the five hypervariable immunogenic regions of HIV-1 gp120 were obtained from the Los Alamos National Laboratory for Human Retroviruses and AIDS, http://www.hiv.lanl.gov/content/immunology. The possible amino acids along the variable epitopes were determined from sequence information of viral isolates. In addition, HLA alleles that could bind to an epitope within HIV HECs were predicted based on HLA Ligand and Motif Database funded by NIH (Sathiamurthy et al., 2003).

HIV HEC peptides representing the five hypervariable regions of gp120 were synthesized by 9fluorenylmethyloxycarbonyl (Fmoc) chemistry, a solidphase peptide synthesis method, utilizing high capacity $(0.7 \mathrm{mmol} / \mathrm{g})$ Knorr resin (Advanced Chemtech) as described previously (Carlos et al., 2000; Kang et al., 2010). Some amino acid coupling steps in the synthesis of the epitope were performed with a mixture of two amino acids as determined from sequence data. As a result, in a single synthesis, a HEC consisting of a mixture of peptides representing the major observed and predicted in vivo variants of the epitope was produced. Each HEC contains 4, 6 or 32 variants of antigenic epitopes of hypervariable regions of gp120. Details of the preparation of HECs and analogs were described in a previous report (Carlos et al., 2000). Single sequence peptides (analogs), representing the sequences found on HIV-1 envelope glycoprotein (gp120) of MN, RF and SF2 strains were also synthesized.

\section{Plasma Collection from Long-Term Non- Progressors}

Approximately $40-50 \mathrm{ml}$ of blood (heparin coated tubes) was obtained by venipuncture from six LTNP individuals every two months over a course of 10 months. Three patient samples were collected at the Dominican Republic (two females and one male) and three in Puerto Rico (one female and two males). All six individuals have had documented HIV infection for 8-14 years and were asymptomatic with no opportunistic infections. Samples were collected under a protocol approved by the corresponding Institutional Review Board (IRB) in the Dominican Republic and Puerto Rico. All subjects gave written informed consent. These individuals were not receiving antiretroviral therapy.

\section{Enzyme-Linked Immunosorbent Assay (ELISA)}

The ELISA procedure was previously described (Kang et al., 2010). All of the plasma samples from HIV LTNPs were heat-inactivated at $56^{\circ} \mathrm{C}$ for $30 \mathrm{~min}$ before testing. Antigens used were the five HIV HECs, a set of HEC analogs representing the sequences found on HIV1 envelope glycoprotein (gp120) of MN, RF and SF2 strains, or recombinant protein gp120 (RF strain). Flatbottomed microtiter plates (Corning Costar) were coated with HIV HECs and analog peptides at a concentration of $5 \mu \mathrm{g} /$ well in triplicate at $37^{\circ} \mathrm{C}$ overnight; Recombinant gp120 protein was plated at a concentration of $1 \mu \mathrm{g} /$ well. $10 \%$ non-fat milk was added to block non-specific binding for $2 \mathrm{~h}$ at $37^{\circ} \mathrm{C}$ and LTNP plasma was added at a $1: 100$ dilution in a volume of $50 \mu \mathrm{l}$ per well. HIVuninfected normal human serum was used as negative control. Antigen-bound antibodies were detected using anti-human IgG conjugated to Horseradish Peroxidase (HRP) at a 1:2000 dilution. SureBlue TMB solution was used as substrate (KPL). The O.D. was measured spectrophotometrically at $650 \mathrm{~nm}$ with an automatic plate reader (VERS Amax, Molecular Devices) according to the manufacturer's instructions (SOFTmax PRO, Molecular Devices).

\section{Statistical Analysis}

The significance of difference in antibody responses between LTNPs and normal human serum was determined using anunpaired Student's t-test. Data were regarded as statistically significant at the two-tailed $\mathrm{p}$ values of $<0.05$. 


\section{Result}

Plasma samples from three LTNP patients were obtained in Puerto Rico (PR) and another three samples in the Dominican Republic (DR).

To determine the extent and breadth of antibody binding to the HIV HECs, we performed ELISA using plasma from LTNPs. We also tried to determine whether the antibody response to HIV gp120 hypervariable regions changes over a 10 -month period by examining two cohorts of three LTNPs (asymptomatic to AIDS and without opportunistic infections) from two different countries (Puerto Rico and the Dominican Republic).

All plasma samples from the six LTNPs showed positive antibody response to all of the individual HIV gp hypervariable regions over the course of the study, except for month 4 or 8 in patients PR1, PR3 and DR3 (Fig. 1). HIV negative, Normal Human Sera (NHS) were obtained from commercial sources or from HIV uninfected healthy individuals and used as a negative control. All six LTNP patients showed the strongest antibody response to HIV HEC 3 among all HIV HECs examined $(p<0.001)$ and the response was as high as or even greater than that obtained against recombinant gp $120(\mathrm{p}<0.001)$. Moreover, the antibody response to HIV HEC 3 remained high during the course of study, showing no significant changes (Fig. 1). The antibody response to HIV HEC 1 was the second highest overall. Interestingly, one of the LTNP patients examined (DR1) showed broadly reactive, strong antibody response to all of the individual HIV HECs and this patient maintained very low viremia over 4 years of follow-up (Fig. 1).

During the same period, we tested antibody responses to monovalent peptides (analogs) representing the same regions of gp120 as the HECs. The results one another, the antibody binding to HIV HECs was greater than to the analogs, including the sequences found in HIV-1 RF, SF2 and MN (Fig. 3). Similar results were observed for patient DR2, although the antibody response to the HIV HECs was not significantly higher than that to analogs except in months 0 and 2 (data not shown).

Patient DR3, with the highest viremia and the lowest ratio of CD4 to CD8 among all the patients examined, showed that regardless of the HIV-1 strain, the average antibody responses of LTNPs to analog epitopes of the V3 region of gp120 were the highest, compared to those to other analog epitopes (Fig. 4-6). Interestingly, this antibody response was weaker than to HIV HEC $3(\mathrm{p}<$ 0.001) (Fig. 2). In addition, when the antibody responses of patient DR1 to different antigens were compared to one another, the antibody binding to HIV HECs was greater than to the analogs, including the sequences found in HIV-1 RF, SF2 and MN (Fig. 3). Similar results were observed for patient DR2, although the antibody response to the HIV HECs was not significantly higher than that to analogs except in months 0 and 2 (data not shown).
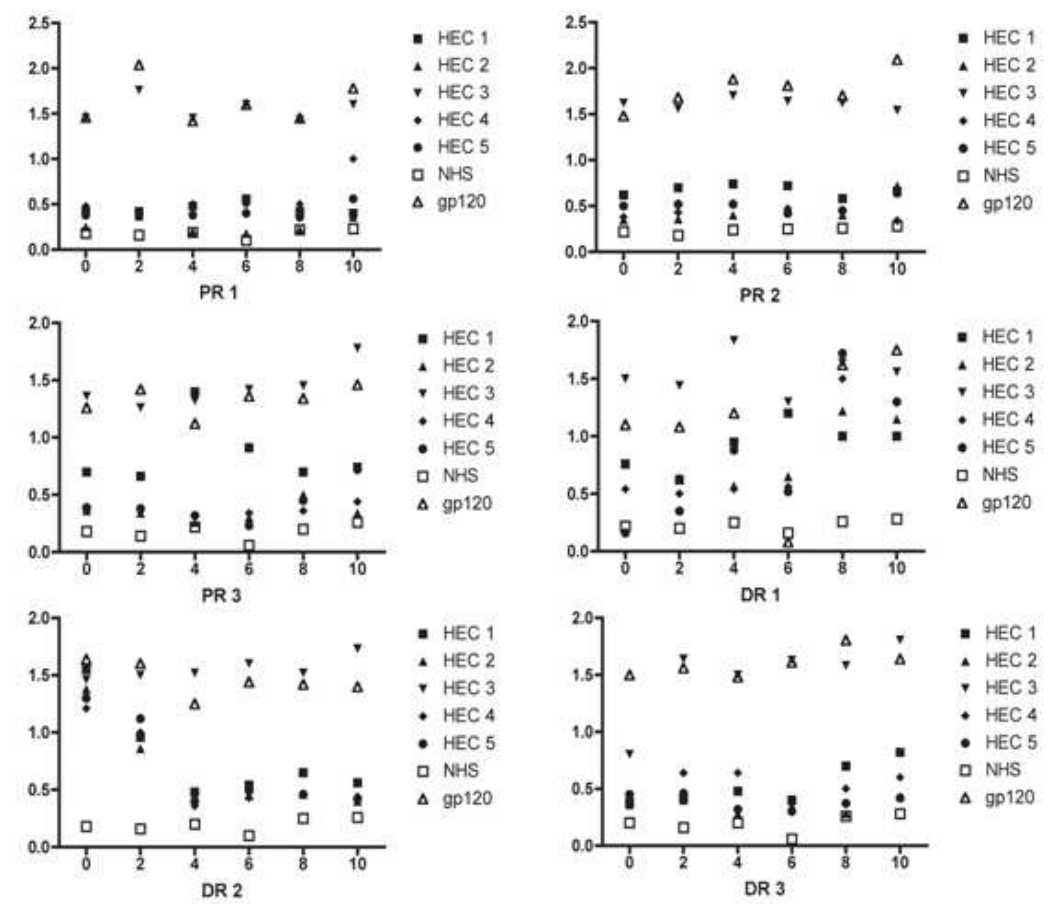

Fig. 1. Immune response of LTNPs to all HIV HECs at $0,2,4,6,8$ and 10 months. All plasma samples from the six LTNPs showed positive antibody response to all of the individual HIV HECs over the course of the study, except for month 4 or 8 in patients PR1, PR3 and DR3. All six LTNP patients showed the strongest antibody response to HIV HEC 3 and HIV HEC 1 was the second highest. NHS: HIV-uninfected normal human serum. Gp120: Positive control. PR1, PR2, PR3: Plasma samples from three LTNP 

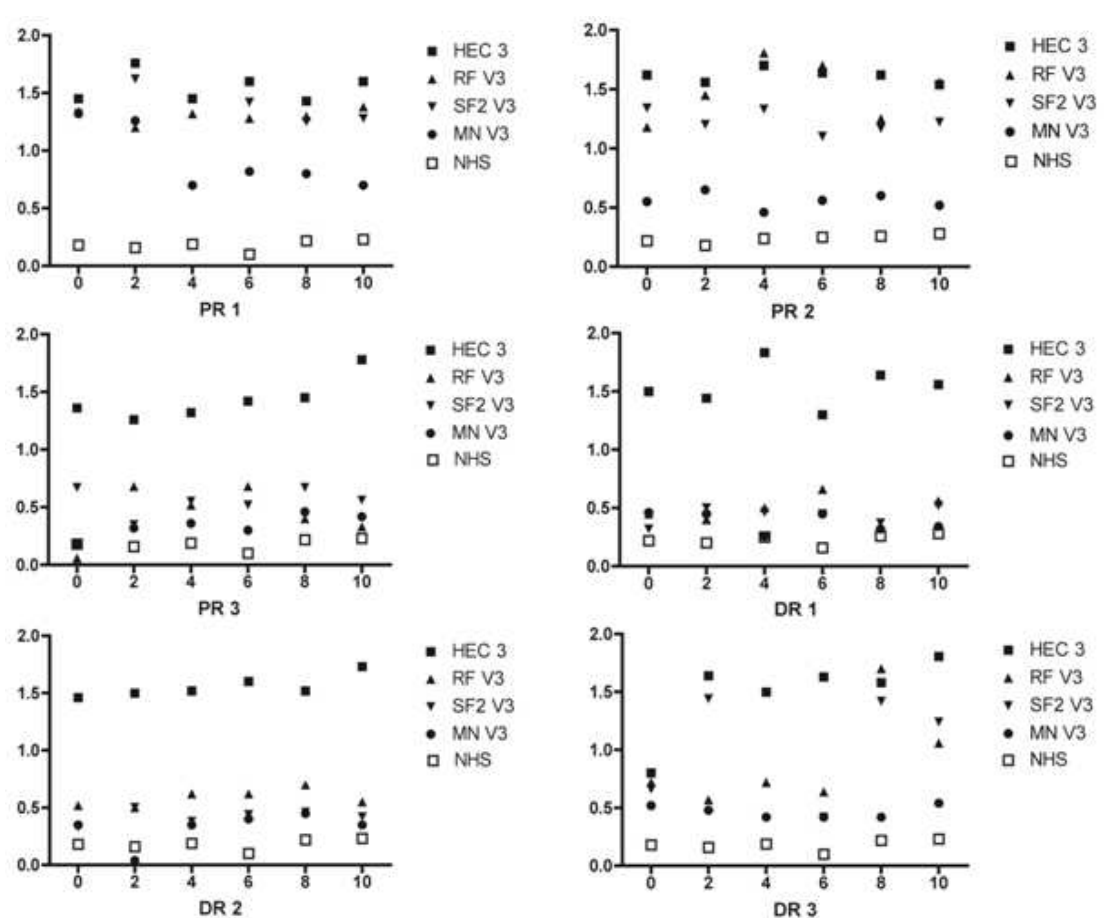

Fig. 2. Antibody responses to gp $120 \mathrm{~V} 3$ region in $0,2,4,6,8,10$ months. All plasma samples from the six LTNPs showed positive antibody response to Gp120 V3 region, but weaker than to HIV HEC 3. MN V3, RF V3, SF2 V3: Single sequence peptides (analogs), representing the V3 sequences found on HIV-1 envelope glycoprotein (gp120) of MN, RF and SF2 strains. NHS: HIV-uninfected normal human serum, as negative control. PR1, PR2, PR3: Plasma samples from three LTNP patients from Puerto Rico; DR1, DR2, DR3: Plasma samples from three LTNP patients from Dominican Republic. Values in the Y axis represent optical density
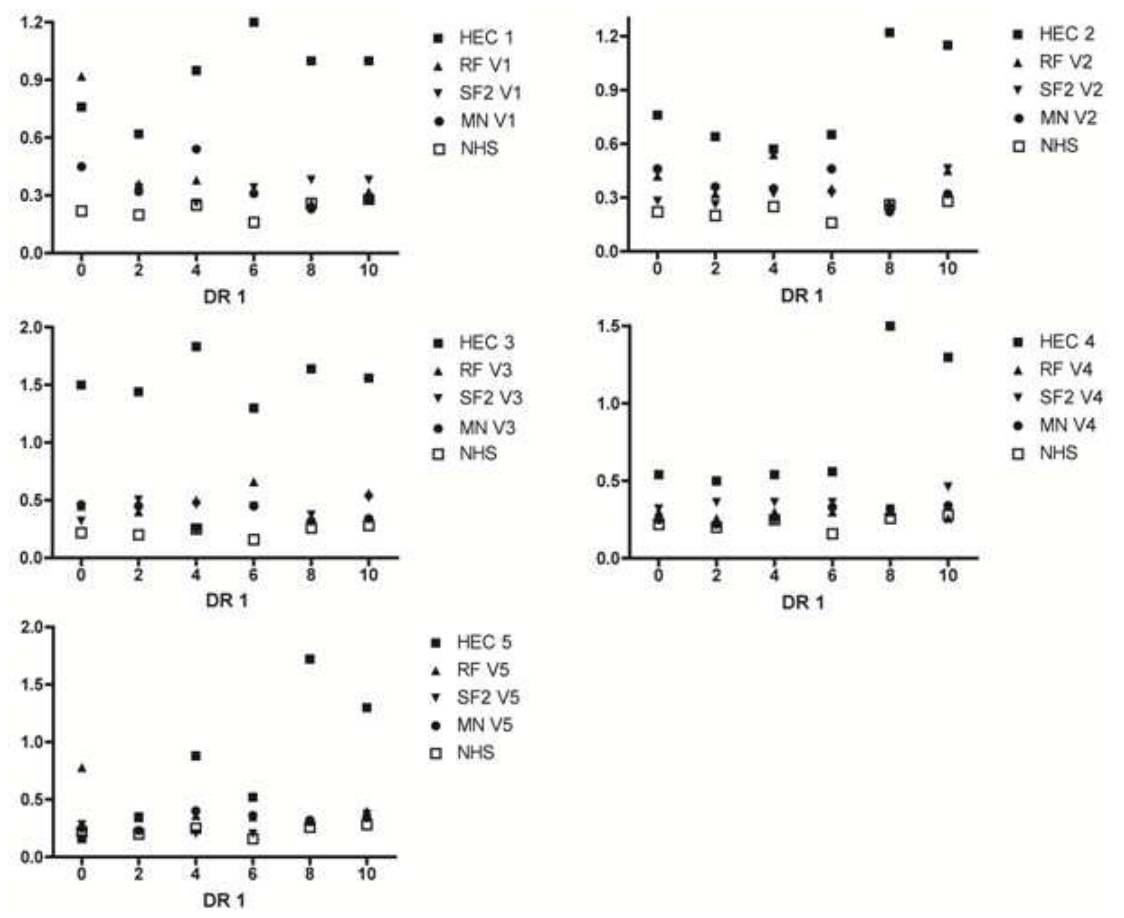

Fig. 3. Antibody responses of patient DR1 in $0,2,4,6,8,10$ months. Plasma sample from patient DR1 showed different level of antibody responses to the analogs of gp120 V1 to V5 regions, the antibody binding to HIV HECs was greater than to the analogs, including HIV-1 RF, SF2 and MN. Values in the Y axis represent optical density 

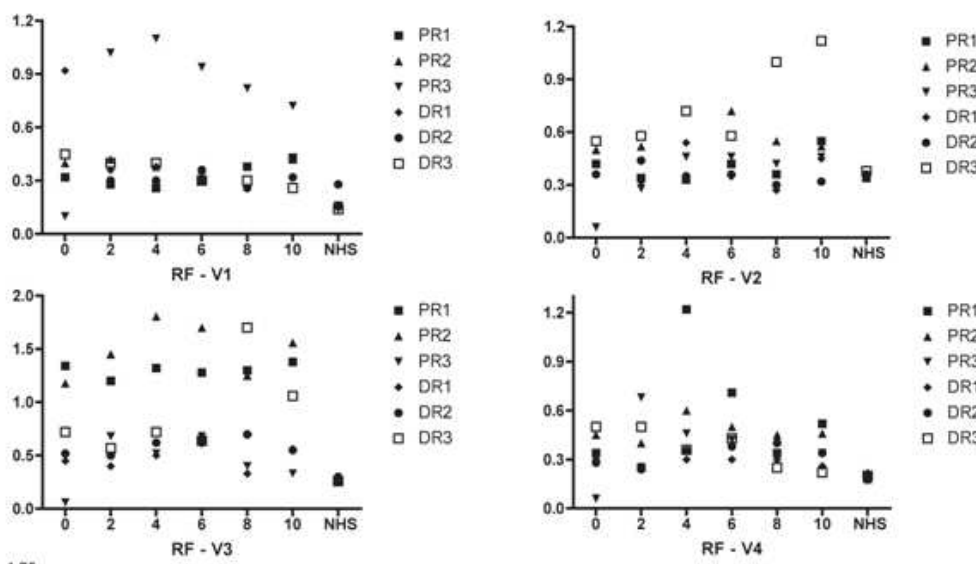

- PR1

$P R 2$
$P R 3$

DR1

- DR2
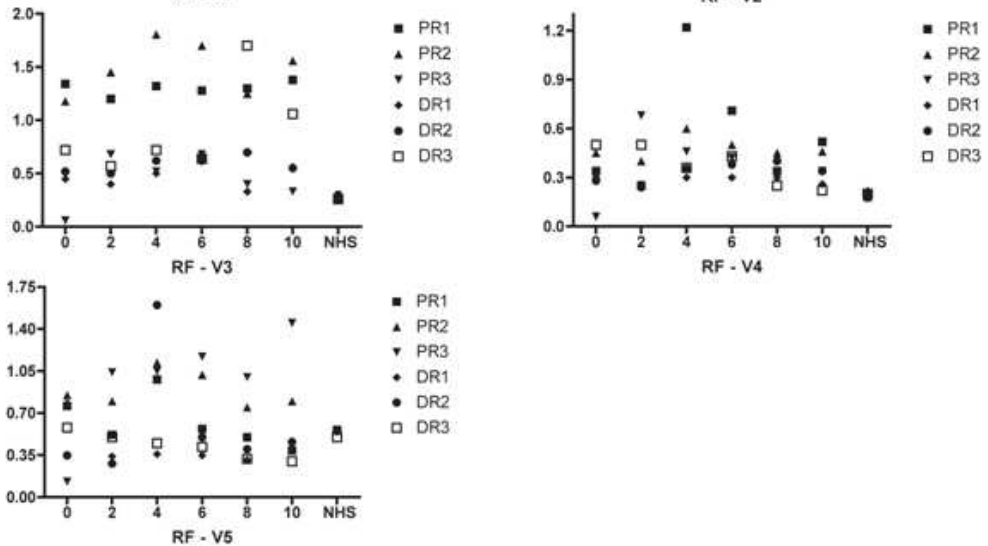

PR1

PR3

DR3

RF - V5

Fig. 4. Antibody responses to gp120 V1 to V5 of HIV1 RF strain in 0, 2, 4, 6, 8, 10 months. NHS: HIV-uninfected normal human serum. Values in the $\mathrm{Y}$ axis represent optical density
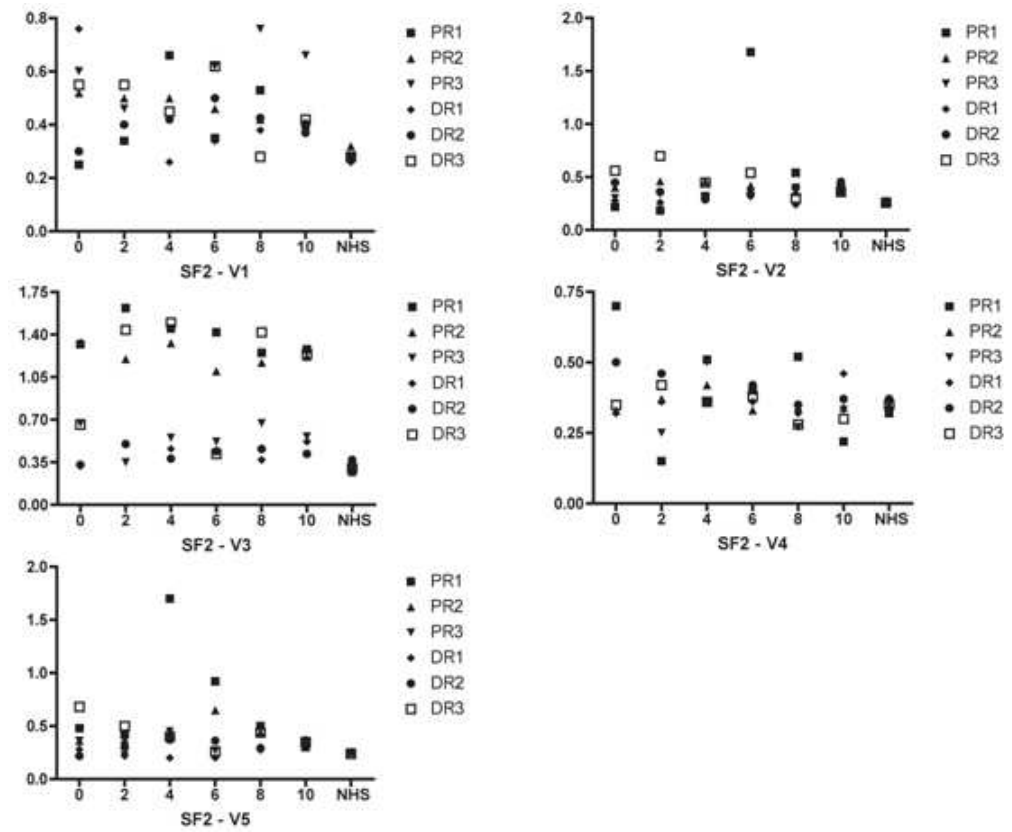

Fig. 5. Antibody responses to gp120 V1 to V5 of HIV1 SF2 strain in 0, 2, 4, 6, 8, 10 months. NHS: HIV-uninfected normal human serum. Values in the $\mathrm{Y}$ axis represent optical density

Patient DR3, with the highest viremia and the lowest ratio of CD4 to CD8 among all the patients examined, had a strong antibody response to the V2 epitope represented by all three analogs tested. PR3 showed a strong antibody response to the V1 epitope of analogs SF2 and RF, but not to MN, which are X4R5 dual tropic and $\mathrm{X} 4$ tropic HIV-1 viruses, respectively. Another interesting finding was that the antibody response to all three analogs of the V3 epitope from patients PR3 and DR1 was relatively lower than the responses of patients PR1, PR2 and DR3 considering that PR3 and DR1 showed the lowest viremia among the patients examined. Patient PR1 showed a transient, strong antibody response to epitopes from V2, V4 and V5, but we could not find any correlation between these antibody responses and viremia or the ratio of CD4 to CD8 (Fig. 4-6). 

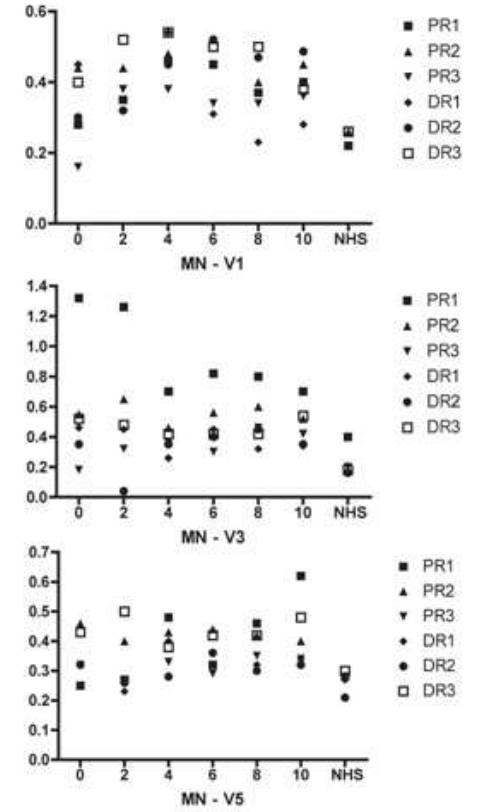
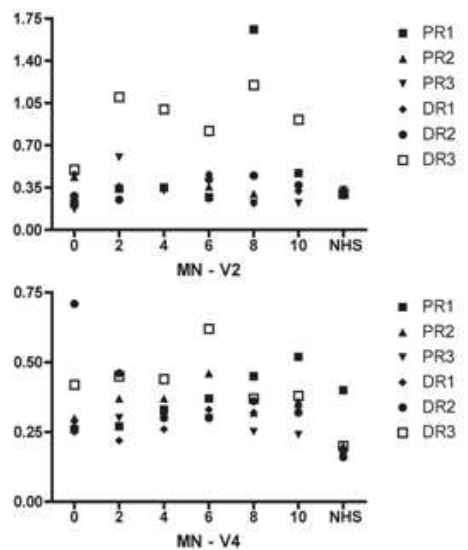

Fig. 6. Antibody responses to gp120 V1 to V5 of HIV1 RF strain at 0, 2, 4, 6, 8 and 10 months. NHS: HIV-uninfected normal human serum. Values in the $\mathrm{Y}$ axis represent optical density

\section{Discussion}

HECs was stronger than to the monovalent peptides that represent single strains of HIV-1 (analogs). It is apparent from the present longitudinal study of a few LTNP patients that these individuals mount a humoral immune response that includes antibodies specific to the five variable epitopes studied. Probably due in part to the different genetic HLA background of each patient, the recognition of HIV HECs and analog epitopes is highly variable and unique.

V3 sequence variability affects virus tropism by determining which co-receptor, CXCR4 or CCR5, is NHS: HIV-uninfected normal human serum. Values in the $\mathrm{Y}$ axis represent optical density.

This study was performed with HIV-infected individuals that were nor receiving antiretroviral therapy. It was surprising to observe that antibodies from all the LTNPs examined reacted to each of the individual HIV HECs and that the overall antibody response to HIV used by HIV to infect target cells (Jensen and van't Wout, 2003) (Jensen et al., 2003). In addition, amino acid substitutions in the V3 loop are associated with the switch from Non-Syncytium-Inducing (NSI) variants to Syncytium-Inducing (SI) variants (Wang et al., 2000; EdoMatas et al., 2011). A disulfide bond formed by two cysteine residues flanking the V3 sequence generates a loop conformation and the tip of the V3 loop consists of a conserved amino acid sequence ( $\mathrm{Su}$ et al., 2000; Huang et al., 2005). Several studies have demonstrated that antibodies elicited against hypervariable and conserved epitopes in the V3 loop showed broadly- reactive neutralization to multiple subtypes of HIV-1 (Zolla-Pazner, 2004; Hioe et al., 2010; Swetnam et al., 2010). In the present study, we also observed that antibody responses to HIV HEC 3 and HEC analog V3 were the strongest, compared to those to other HECs and analogs. However, we did not test to what extent and breadth the antibodies that reacted to HIV HEC V3 were involved in the induction of cross-reactive neutralization to variable or conserved epitopes.

For the patients studied here, we do not know the sequence of the infecting virus; thus, a correlation between the epitopes represented in the viral strain and the patients' response could not be assessed at this time. Moreover, the antibody responses from individual LTNP patients were extremely variable to different antigens, including HIV HECs and analog peptides, HIV-1 RF, SF2 and MN, during the time period examined. This extreme variability in the antibody responses of individual LTNP patients made it difficult to find any correlation between antibody responses and viremia or CD4:CD8 ratios. However, it is very likely that each person was infected by a different strain or a different collection of strains. This may contribute to the stronger antibody response to HIV HECs than to analogs representing single strains of HIV-1. As samples from truly untreated LTNPs are very hard to obtain, these studies only provide a small window into the immunological, virological and clinical parameters documented over time in these unique patients. Further studies with a larger number of patient samples is needed to determine whether patients with low viremia have strong antibody responses to the V2 epitope of 
gp120, or have stronger antibody responses to HIV HECs (variable epitopes) than patients with high viremia. Interestingly, two genetic signatures at two amino acid positions in V2 were found to be less common in individuals infected after vaccination with the RV144 vaccine (Rolland et al., 2012). Additional information concerning $\mathrm{T}$ helper, cytotoxic and suppressor responses needs to be obtained to further understand the correlation between the immune responses to HIV gp120 hypervariable epitopes and the long delay to progression to AIDS observed in LTNPs.

HIV HECs represent highly variable antigenic epitopes from all five variable regions of the HIV-1 envelope glycoprotein 120. Barouch et al. (2010) demonstrated that a bivalent mixture of mosaic HIV-1 proteins consisting of two HIV-1 Gag, Pol and Env antigens elicited stronger cellular immune responses to variant $\mathrm{T}$ cell epitope peptides in rhesus macaques than those of naturally occurring or consensus antigens (Barouch et al., 2010). This experimental design is similar to that employed in our previous studies (Carlos et al., 1999; 2000; Azizi et al., 2008; Kang et al., 2010), though Barouch et al. (2010) used antigenic epitopes containing more limited variability in sequence polymorphism.

The fact that antibodies from some LTNPs reacted more strongly to individual HIV HECs than to single strain peptides proves that HIV HECs enhance the sensitivity of recognition of antibodies elicited by HIV-1 infection. This information could contribute to the development of an immunogen to be included in the design of preventive and therapeutic vaccine approaches against HIV-1 infection and AIDS.

\section{Acknowledgement}

This work was partially supported by National Cancer Institute (5F31CA94833-02).

\section{Author's Contributions}

Rebecca Rivera and Kyung Hee Kang: Performed experiments and data interpretation. Participated in manuscript preparation.

Murray B. Gardner and David E. Anderson: Performed data interpretation. Participated in manuscript preparation.

Santiago Collado-Chastel, Eddy Rios-Olivares and Yasuhiro Yamamura: Collected, categorized and provided clinical samples.

Francisco Diaz-Mitoma: Performed data interpretation.

Xia Li: Performed data interpretation. Participated in manuscript revision.

José V. Torres: Planed the project and directed the research group.

\section{Ethics}

This article is original and contains unpublished material. The corresponding author confirms that all of the other authors have read and approved the manuscript and no ethical issues involved.

\section{References}

Azizi, A., D.E. Anderson, J.V. Torres, A. Ogrel and M. Ghorbani et al., 2008. Induction of broad crosssubtype-specific HIV-1 immune responses by a novel multivalent HIV-1 peptide vaccine in cynomolgus macaques. J. Immunol., 180: 2174-2186. PMID: 18250424

Barouch, D.H., K.L. O'Brien, N.L. Simmons, S.L. King and P. Abbink et al., 2010. Mosaic HIV-1 vaccines expand the breadth and depth of cellular immune responses in rhesus monkeys. Nat Med., 16: 319-323. DOI: $10.1038 / \mathrm{nm} .2089$

Cardozo, T., J. Swetnam, A. Pinter, C. Krachmarov and A. Nadas et al., 2009. Worldwide distribution of HIV type 1 epitopes recognized by human anti-V3 monoclonal antibodies. AIDS Res. Hum Retroviruses, 25: 441-450. DOI: 10.1089/aid.2008.0188

Carlos, M.P., D.E. Anderson, M.B. Gardner and J.V. Torres, 2000. Immunogenicity of a vaccine preparation representing the variable regions of the HIV type 1 envelope glycoprotein. AIDS Res. Hum Retroviruses, 16: 153-161. DOI: 10.1089/088922200309494

Carlos, M.P., Y. Yamamura, F. Díaz-Mitoma and J.V. Torres, 1999. Antibodies from HIV-positive and AIDS patients bind to an HIV envelope multivalent vaccine. J. Acquir Immune Defic. Syndr., 22: 317-324. PMID: 10634192

Curlin, M.E., R. Zioni, S.E. Hawes, Y. Liu and W. Deng et al., 2010. HIV-1 envelope subregion length variation during disease progression. PLoSPathog.

Edo-Matas, D., K.A. van Dort, L.C. Setiawan, H. Schuitemaker and N.A. Kootstra, 2011. Comparison of in vivo and in vitro evolution of CCR5 to CXCR4 coreceptor use of primary human immunodeficiency virus type 1 variants. Virology, 412: 269-277. DOI: 10.1016/j.virol.2011.01.010

Feng, Y., C.C. Broder, P.E. Kennedy and E.A. Berger, 1996. HIV-1 entry cofactor: functional cDNA cloning of a seven-transmembrane, $G$ proteincoupled receptor. Science, 272: 872-877. DOI: $10.1126 /$ science. 272.5263 .872

Fomsgaard, A., H.V. Nielsen, K. Bryder, C. Nielsen and R. Machuca et al. 1998. Improved humoral and cellular immune responses against the gp120 V3 loop of HIV-1 following genetic immunization with a chimeric DNA vaccine encoding the V3 inserted into the hepatitis B surface antigen. Scand J. Immunol., 47: 289-295. PMID: 9600309 
Helseth, E., U. Olshevsky, C. Furman and J. Sodroski, 1991. Human immunodeficiency virus type 1 gp120 envelope glycoprotein regions important for association with the gp41 transmembrane glycoprotein. J. Virol., 65: 2119-2123. PMID: 2002555

Hioe, C.E., T. Wrin, M.S. Seaman, X. Yu and B. Wood et al., 2010. Anti-V3 monoclonal antibodies display broad neutralizing activities against multiple HIV-1 subtypes. PLoS One, 5: e10254-e10254.

Huang, C.C., M. Tang, M.Y. Zhang, S. Majeed and E. Montabana et al., 2005. Structure of a V3containing HIV-1 gp120 core. Science, 310: 1025-1028. DOI: $10.1126 /$ science. 1118398

Hughes, E.S. and J.E. Bell, 1997. Investigation of population diversity of human immunodeficiency virus type 1 in vivo by nucleotide sequencing and length polymorphism analysis of the V1/V2 hypervariable region of env. J. Gen Virol., 78: 2871-2882. DOI: 10.1099/0022-1317-78-11-2871

Jensen, M.A., F.S. Li, A.B. van't Wout, D.C. Nickle and D. Shriner et al., 2003. Improved coreceptor usage prediction and genotypic monitoring of R5-to-X4 transition by motif analysis of human immunodeficiency virus type 1 env V3 loop sequences. J. Virol., 77: 13376-13388.

DOI: $10.1128 /$ JVI.77.24.13376-13388.2003

Jensen, M.A. and A.B. van 't Wout 2003. Predicting HIV-1 coreceptor usage with sequence analysis. AIDS Rev., 5: 104-112. PMID: 12876899

Kang, K.H., Y. Yamamura, M.P. Carlos, N. Karvelas and I.S. Kim et al., 2010. Synthetic antigens representing the antigenic variation of human hepatitis C virus. Viral Immunol., 23: 497-508. DOI: $10.1089 /$ vim. 2010.0043

Kitrinos, K.M., N.G. Hoffman, Julie A.E. Nelson and R. Swanstrom, 2003. Turnover of env variable region 1 and 2 genotypes in subjects with late-stage human immunodeficiency virus type 1 infection. J. Virol., 77: 6811-6822. DOI: 10.1128/JVI.77.12.6811-6822.2003

Kwong, P.D., R. Wyatt, J. Robinson, R.W. Sweet and J. Sodroski et al., 1998. Structure of an HIV gp120 envelope glycoprotein in complex with the CD4 receptor and a neutralizing human antibody. Nature, 393: 648-659. DOI: $10.1038 / 31405$

Modrow, S., B.H. Hahn, G.M. Shaw, R.C. Gallo and F. Wong-Staal et al., 1987. Computer-assisted analysis of envelope protein sequences of seven human immunodeficiency virus isolates: Prediction of antigenic epitopes in conserved and variable regions. J. Virol., 61: 570-578.

Pantophlet, R. and D.R. Burton, 2006. GP120: Target for neutralizing HIV-1 antibodies. Annu. Rev. Immunol., 24: 739-769.

DOI: 10.1146/annurev.immunol.24.021605.090557
Rolland, M., P.T. Edlefsen, B.B. Larsen, S. Tovanabutra and E. Sanders-Buell et al., 2012. Increased HIV-1 vaccine efficacy against viruses with genetic signatures in Env V2. Nature, 490: 417-420. DOI: 10.1038 /nature 11519

Sagar, M., X. Wu, S. Lee and J. Overbaugh, 2006. Human immunodeficiency virus type 1 V1-V2 envelope loop sequences expand and add glycosylation sites over the course of infection and these modifications affect antibody neutralization sensitivity. J. Virol., 80: 9586-9598.

DOI: $10.1128 / J V I .00141-06$

Sathiamurthy, M., H.D. Hickman, J.W. Cavett, A. Zahoor and K. Prilliman et al., 2003. Population of the HLA ligand database. Tissue Antigens, 61: 12-19. DOI: $10.1034 /$ j.1399-0039.2003.610102.x

Shioda, T., S. Oka, X. Xin, H. Liu and R. Harukuni et al., 1997. In vivo sequence variability of human immunodeficiency virus type 1 envelope gp120: Association of V2 extension with slow disease progression. J. Virol., 71: 4871-4881. PMID: 9188549

Speck, R.F., K. Wehrly, E.J. Platt, R.E. Atchison and I.F. Charo et al., 1997. Selective employment of chemokine receptors as human immunodeficiency virus type 1 coreceptors determined by individual amino acids within the envelope V3 loop. J. Virol., 71: 7136-7139. PMID: 9261451

Su, J., A. Palm, Y. Wu, S. Sandin and S. Hoglund et al., 2000. Deletion of the GPG motif in the HIV type 1 V3 loop does not abrogate infection in all cells. AIDS Res. Hum Retroviruses, 16: 37-48. DOI: $10.1089 / 088922200309584$

Swetnam, J., E. Shmelkov, S. Zolla-Pazner and T. Cardozo, 2010. Comparative magnitude of cross-strain conservation of HIV variable loop neutralization epitopes. PLoS One.

DOI: 10.1371/journal.pone.0015994

Wang, W.K., C.N. Lee, T. Dudek, S.Y. Chang and Y.J. Zhao et al., 2000. Interaction between HIV type 1 glycoprotein 120 and CXCR4 coreceptor involves a highly conserved arginine residue in hypervariable region 3. AIDS Res Hum Retroviruses, 16: 1821-1829. DOI: $10.1089 / 08892220050195784$

Wyatt, R. and J. Sodroski, 1998. The HIV-1 envelope glycoproteins: Fusogens, antigens and immunogens. Science, 280: 1884-1888.

DOI: $10.1126 /$ science. 280.5371 .1884

Zolla-Pazner, S., 2004. Identifying epitopes of HIV-1 that induce protective antibodies. Nat Rev. Immunol., 4: 199-210. DOI: 10.1038/nri1307 